Bangladesh Journal of Bioethics 2017; 8(2):19-22

\title{
Ethical Justification of Involving Human Volunteers in Phase 1 Trials
}

\section{Zoheb Rafique}

Lecturer Department of Community Medicine, Liaquat University of Medical and Health Sciences (LUMHS), Jamshoro Sindh. Email: scorpionzoheb@hotmail.com

Introduction: Tremendous development in recent medical science and the consequent discoveries resulting in successful prevention and also cure of different diseases are shared by clinical research involving the human volunteers. Preceding the trials in the human subjects, and to ensure safety, the proposed drug and other interventions are either tested in animals (vivo) or in laboratory (vitro) to evaluate initial safe starting dose for the human beings and to key out the benchmarks for the clinical monitoring for the potential unfavorable effects. These pre human trials might not necessarily protect against the untoward effects in the human beings as happened in the case of thalidomide tragedy, which caused disability and killed thousands of babies born to the mothers, those who took this medicine. Use of healthy human volunteers in the preliminary experiments or phase I clinical trials either reduces or excludes risks of subsequent undesirable effects in the future trails (1). Phase-1 trials are conducted in order to test the safety, reactions and immunogenicity of vaccines in volunteers. Novel treatments for the cancer are first tested in phase 1 trials enrolling the patients with advanced disease, who have exhausted the standard treatment options. Phase-1 oncology trials are the pivot point in the translation of new cancer therapies from bench to bedside. Nevertheless, these trials remain ethically controversial. The controversy stems from the fact that, classically, phase-1 oncology clinical trials involve first-inhuman testing of experimental treatment candidates in patients with a terminal diagnosis, who typically have exhausted standard treatment options. Commentators on the ethics of phase-1 clinical trials make diametrically opposed claims about the prospect of direct medical benefit from participation in these trials-benefits that can be attributed to receiving the experimental treatment intervention. One camp of benefit skeptics, inhabited mainly by bioethicists, characterizes this form of research as lacking any reasonable prospect of direct medical benefit. They see an ethical cloud hovering over phase-1 trials, because the vast majority of patients volunteer for phase- 1 trials out of a motivation to receive medical benefit. In the view of these skeptics, such patients therefore harbor a 'therapeutic misconception' about research participation. This misconception calls into question the validity of informed consent and thereby undercuts the ethical basis of these trials (2). In this paper, I will discuss the ethical justification of the participation of human volunteers in phase- 1 trials.

Key words: Ethical Justification, Human Volunteers, Phase 1 Trials

Discussion: It is now widely accepted that medical research designed for the benefit of populations in developed countries should not be conducted with subjects recruited from populations in economically underdeveloped countries (3). Indeed, it is ethically objectionable to recruit from populations in resource-poor settings, even in developed countries, unless those 
populations are particularly susceptible to the condition the research is designed to relieve. In one study, there was proposal to conduct a phase-1 vaccine study recruiting subjects from the United States when the purpose is to assist the population of Mali, in Sub-Saharan Africa, to overcome the pervasive local consequences of Malaria. The ethical principle of justice, which requires a fair allocation of the risks and benefits of medical research, provides that the risk of research should not be planned to affect subjects from one population when benefits of the research are primarily directed to another population. It may accordingly appear, at first assessment, that the Malian government's requirement that all phase- 1 testing of the antimalarial vaccine be conducted in the United States is as unethical as it would be for the United States government to require that all phase- 1 testing of a vaccine or other product intended primarily to benefit the population of the United States be conducted in Mali. Yet codes of ethical conduct are less consistent on this point than commentators usually require being. The World Medical Association's much-cited Declaration of Helsinki: Ethical principles for Medical Research Involving Human subjects provides that "Medical research is only justified if there is a reasonable likelihood that the population in which the research is carried out stand to benefit from the results of the research." By this criterion, conduct of the phase-1 study in the United States appears unethical. In the context of HIV/AIDS, however, the WHO'S Global program on AIDS (1989) provided that, "in general, initialphase-1 trials should be conducted in the country of origin of the vaccine". By this criterion, phase-1 testing in the United States is appropriate, if a vaccine would originate and initially be governmentally approved in the United States. The situation would be otherwise, of course, if the NIH was funding the study for production of the vaccine in Mali. The ethical remains of where the initial phase-1vaccine trials can be conducted most equitably, with least risk of exploitation and most protection of the interests of study subjects (4). Most clinical research trials today require the informed consent. Concern however is raised that subjects of phase 1 trial studies might not provide valid consent. In particular, few commentators worry that subjects of phase 1 oncology trials have an exaggerated idea of any chance of the therapeutic benefit. The Empirical studies tells that phase 1 trial participants are highly optimistic and hopeful about their chance of personal benefit and also are motivated by hope for the clinical improvement. Altruism, on other hand is much less often identified as driving the decision to enroll, when quoted as motivating factor; and it typically is not the prime reason for the participation. In one research study, sixty one $(61 \%)$ of phase 1 oncology participants were doubtful about altruism would motivate the advanced cancer patients to enroll in the non-beneficial research and several phase 1 volunteers in another study showed "Surprise" at idea of research participation based exclusively on altruism. Some studies find, however that the individuals in some other types of research trials often participate in the hope of helping others (5). As an example, schaeffer and colleagues describe that "hope others benefit" is one of the two most common motivational factors for the healthy volunteers (6). The research participants should be fully informed about the difference between research and therapy and also risk-benefit ratio. The researcher should offer patients substitutes other than participation in the trials and also vulnerable population should not be included in the trials at any cost and 
especially in thephase-1 trials. CIOMS guideline 7 talks about inducement of participation in research. According to the guideline the research subjects can be reimbursed for their needs such as transport and other expenses, and also lost earnings, that is associated with participation in the research. Those persons who receive no any direct benefit from research may also get a small amount of money for their inconvenience due to the participation in research. All volunteers may get the medical services unrelated to research and could have tests and procedures performed free of cost. Payments in terms of money or in kind to the research subjects may not be so huge as to carry them to take unwarranted risks or volunteer against the better judgment. Incompetent persons are vulnerable to the exploitation for financial gain by the guardians. A subject who withdraws himself/herself from the research for various reasons related to research study, such as unacceptable side-effects of a study drug, or who is withdrawn on health grounds, should be paid or recompensed as if full participation had taken place. For all biomedical research involving human subjects, the investigator must ensure that potential benefits and risks are reasonably balanced and risks are minimized. The Declaration of Helsinki deals with the wellbeing of research subjects and the avoidance of risk. Thus, considerations related to the well-being of the human subject should take precedence over the interests of science and society, clinical testing must be preceded by adequate laboratory or animal experimentation to demonstrate a reasonable probability of success without undue risk, every project should be preceded by careful judgment of predictable burden and risks in comparison with the foreseeable benefits to research subjects or to others; physician-researchers must be confident that the risks involved have been adequately assessed and can be satisfactorily managed; and the risks and burdens to the subject must be minimized, and reasonable in relation to the importance of the objective or the knowledge to be gained (7).

Conclusion: the first basic principle of the Declaration of Helsinki requires biomedical research involving human subjects to be based on "adequately performed laboratory and animal experimentation and on a thorough knowledge of the scientific literature." This implies that human subjects should not be used unless and until successful experiments in animals, as well as in vitro, have been completed. The Declaration might allow proceeding to clinical trials if adequate animal studies demonstrated the inapplicability irrelevance, or absence of a useful animal model. If animals died immediately after receiving a vaccine, this would undoubtedly prohibit using the vaccine in human subjects (8). I will conclude this paper by saying that research is the only way of getting rid of various diseases through proper treatment and also benefiting future patients from those diseases which have no cure at present or any medical treatment available, so research should not be stopped. In my opinion, human volunteers can be involved in phase-1 trials but following all international guidelines and all other aspects of biomedical ethics.

Author Contribution: I am the only author of this manuscript

Conflict of Interest: No conflict of interest 


\section{References:}

1. Inayat Ullah Memon. Justification of participation of human subjects in phase 1 clinical trials: an ethical analysis. Bangladesh Journal of Bioethics 2011;2(2):26-29.

2. Franklin G Miller and Steven Joffe. Benefit in phase 1 oncology trials: therapeutic misconception or reasonable treatment option? Clinical Trials. 2008; 5: 617-623.

3. James V. Lavery Et Al. Ethical Issues in International Biomedical Research. A Casebook by Oxford University Press. 2007.

4. Bernard Dickens. Reverse Exploitation in the Baltimore Malaria Vaccine Study. Commentary 11.2: A Casebook by Oxford University Press. 2007.

5. Emily Abdoler, Holly Taylor, and David Wendler. The Ethics of Phase 0 Oncology Trials. Clin cancer Res. 2008; 14(12): 3692-3697.

6. Schaeffer MH, Krantz DS, Wichman A, Masur H, Reed E, Vinicky JK. The impact of disease severity on the informed consent process in clinical research. Am J Med 1996; 100: 261-268.

7. International Ethical Guidelines for Biomedical Research Involving Human Subjects. CIOMS 2002; 1-113.

8. Wendy K. Mariner. Why Clinical Trials of AIDS Vaccines Are Premature. Public Health and the Law. AJPH. 1989; Vol. 79, No. 1: 86-91. 